\title{
INTERSTELLAR GAS AROUND WO STARS IN THE GALAXY AND THE MAGELLANIC CLOUDS
}

\author{
TATIANA A. LOZINSKAYA \\ Sternberg State Astronomical Institute \\ Moscow \\ USSR
}

\begin{abstract}
The four oxygen-sequence WR stars, Sand 1 in the Small Magellanic Cloud (SMC), Sand 2 in the Large Magellanic Cloud (LMC), and WR102 and WR 142 in the Galaxy represent the latest stage of the evolution of massive stars (Sanduleak 1971, Barlow and Hummer 1982, Moffat $e$ t al. 1985). We have shown WR 102 to be a stripped CO core of a supermassive star (Dopita et al. 1990), probably seen only several thousand years before a SN explosion. The four stars are characterized by extremely energetic stellar winds - Vw from 4500 to $7400 \mathrm{~km} / \mathrm{s}$ (Barlow and Hummer 1982, Dopita et al. 1990, Torres et al. 1986). Examination of the environments of WO stars leads to the conclusion that the four objects appear to be associated with optical and/or IR shell-like structures, although the short WO-superwind does not prevail in the shell's formation.
\end{abstract}

\section{The nebula $\mathbf{G 2 . 4 + 1 . 4}$}

The nebula G2.4+1.4 around WR 102 is the only object from the four which was shown to be a classical bubble blown by the wind of WR 102 (Dopita et al. 1990, Dopita and Lozinskaya, 1990). We have determined the bubble's size to be $11 \pm 4 \mathrm{pc}$, and its expansion velocity $48 \pm 3 \mathrm{~km} / \mathrm{s}$, and have explained the unusual morphology of the nebula as a "blister" bubble breaking out of the dense cloud. With the ambient gas densities from $2-4$ to $30-60 \mathrm{~cm}^{-3}$ and $\mathrm{Lw}=(1-2) \times 10^{38} \mathrm{erg} / \mathrm{s}$, the shell's dynamical age $\left(t=(0.9-1.3) \times 10^{5} \mathrm{yrs}\right)$ is much longer than the WO-superwind duration. This indicates that the precursor WR-star has created a ring nebula before the WO-stage. IRAS observations reveal an incomplete ring coincident with the edge of the optical nebula (Graham 1985); see also Lozinskaya 1990).

\section{WR 142}

WR 142 (a member of $\mathrm{Be} 87$, distance $\mathrm{d}=1 \mathrm{kpc}$ ) is located at the edge of a giant molecular cloud (Turner and Forbes 1982, Pitault 1981). There is no prominent optical emission around the star because of heavy absorption. The 60 and $100 \mu$ IRAS images reveal a prominent half-shell of about 50 pc (Lozinskaya 1990, Lozinskaya and Repin 1990). We have calculated that $\mathrm{T}_{\text {dust }}(60 / 100)=29-30 \mathrm{~K}$ and $\mathrm{M}_{\text {gas }}=(1-3) \times 10^{3} \times(\mathrm{d} / 1 \mathrm{kpc})^{2} \mathrm{M}_{\mathrm{o}}$. If the IR-shell contains the gas swept from the interior, then the initial density is $n_{o}=1 \mathrm{~cm}^{-3}$. With $L_{w}=\left(1-2 \times 10^{38} \mathrm{erg} / \mathrm{s}\right.$ the bubble's age is $t=\sim(5-7) \times 10^{4} \mathrm{yrs}$, again leading to the conclusion that the IR-shell has been created by the precursor WR-star and perhaps by the neighbouring WR and Of stars which supply energy to the common shell. 


\section{W04+04V binary Sand 1}

The environments of the WO4+O4V binary Sand 1 (the brightest member of NGC 602c (Westerlund 1964)) seem to be similar to WR 142. NGC 602c is situated inside a faint filamentary supershell, DEM 167 of 35'arc in diameter, while DEM 166, associated with NGC 602a,b, is a bright compact HII region on its edge (Westerlund 1964, Davies et al. 1976, Meaburn 1980). There is an obvious ring of IR emission coinciding with the optical supershell (see the IRAS $100 \mu$ image of SMC in Loiseau et al. 1987). Surely Sand 1 is not the only blue star inside the shell. However, the difference between the two HII regions (the compact one connected with NGC $602 \mathrm{a}, \mathrm{b}$, and the thin ring around NGC 602c) may be considered as indicative of the presence of Sand 1.

NGC 602a,b and NGC 602c form a single association with similar stellar contents (except of Sand 1) and similar masses of associated gas $M(D E M 166)=2 \times 10^{4} M_{\odot}, M($ shell $)=3 \times 10^{4} M_{\odot}$ (Westerlund 1964, Kontizas et al. 1988). For the WR+O4 wind energy output $\mathrm{Lw}=\sim 2 \times 10^{38} \mathrm{erg} / \mathrm{s}$ (from $\left\langle M_{W-R}\right\rangle=\sim 4 \times 10^{-5} \mathrm{M}_{\odot} \mathrm{yr}^{-1}$ (Moffat et al. 1985), and $\mathrm{V}_{\mathrm{W}}=4200 \mathrm{~km} \mathrm{~s}^{-1}$ (Barlow and Hummer 1982)), and $\mathrm{n}_{\mathrm{o}}=0.5-1 \mathrm{~cm}^{-3}$ (from $\mathrm{N}_{\mathrm{H}}=1.2 \times 10^{21}$ atoms cm-2 (McGee and Newton $1981 / 2)$ ), the wind duration required to create a bubble of radius $260-300 \mathrm{pc}$ is $\mathrm{t}=(2.5-5) \times 10^{6} \mathrm{yr}$, which is suitably short for the lifetime of such a massive system.

\section{Sand 2}

Sand 2 in the LMC appears to be superimposed on the faint diffuse filamentary HII region DEM 268 (Davies et al. 1976) with a smaller filamentary ring comprising the WO star of about 20'arc (270 pc); a deep-sky photograph outlines a very weak supershell as large as $\sim 1 \mathrm{deg}$. (Meaburn 1980). Detailed observations are required to reveal the nature of the filaments around Sand 2 more fully.

\section{References}

Barlow, M.J. and Hummer, D.G. (1982), IAU Symp. 99 Wolf-Rayet Stars: Observations, Physics, Evolution, C.W.H. de Loore, A.J. Willis (eds). p. 387.

Davies, R.D., Elliott, K.H. and Meaburn, J. (1976), Mem. R. Astron. Soc. 81, 89.

Dopita, M.A., Lozinskaya, T.A., McGregor, P.J. and Rawlings, S.J. (1990), Ap. J. 351, 563.

Dopita, M. and Lozinskaya, T.A. (1990), Ap. J. in press.

Graham, J.R. (1985), Observatory. 105, 7.

Kontizas, E., Morgan, D.H., Kontizas, M. and Dapergolas, A. (1988), A. Ap. 201, 208.

Loiseau, N., Klein, U., Greibe, A., Wielebinski, R. and Haynes, R.F. (1987), A. Ap. 178, 62.

Lozinskaya, T.A. (1990), IAU Symp. 143. in press.

Lozinskaya, T.A. and Repin, S.V. (1990), Sov. Astron. J. in press.

Meaburn, J. (1980), M.N.R.A.S. 192, 365.

Moffat, A.F.J., Breysacher, J. and Seggewiss, W. (1985), Ap. J. 292, 511.

McGee, R.X. and Newton, L.M. (1981/2), Proc. Astron. Soc. Austral. 4, 189 \& 308.

Pitault, A. (1981), A. Ap. 97, L5.

Sanduleak, N. (1971), Astrophys. J. 164, L71.

Torres, A.V., Conti, P.S. and Massey, P. (1986), Ap. J. 300, 379.

Turner, D.G. and Forbes, D. (1982), Publ. Astron. Soc. Pac. 94, 789.

Westerlund, B.E. (1964), M.N.R.A.S. 127, 429. 